\title{
Erratum: Modeling all-electrical detection of the inverse Edelstein effect by spin-polarized tunneling in a topological-insulator/ferromagnetic-metal heterostructure [Phys. Rev. B 97, 144417 (2018)]
}

Rik Dey, Leonard F. Register, and Sanjay K. Banerjee

\author{
(Received 28 November 2018; published 26 December 2018)
}

DOI: 10.1103/PhysRevB.98.219908

We have found the following typographical errors in the original article. The function $\mu_{Y}$ is a function of $y$ and should $\operatorname{read} \mu_{Y}=B_{1} e^{s_{1} y}+B_{2} e^{s_{2} y}$ instead of $\mu_{Y}=B_{1} e^{s_{1} x}+B_{2} e^{s_{2} x}$. Hence, $j_{y}$ should read $j_{y}=-\sigma^{\prime} c_{Y}\left(B_{1} e^{s_{1} y}-B_{2} e^{s_{2} y}\right) \mu_{X}(x)$ instead of $j_{y}=-\sigma^{\prime} c_{Y}\left(B_{1} e^{s_{1} x}-B_{2} e^{s_{2} x}\right) \mu_{X}(x)$. In the same paragraph, the sentence "The solution of the boundary condition $j_{x}=$ $0, \forall y$ at $x=0, L_{x}$ implies $r_{1}=r_{2}=b_{0} m_{y}$, i.e., $c_{X}=0, c_{Y}=c_{0}$." should read "The solution of the boundary condition $j_{x}=$ $0, \forall y$ at $x=0, L_{x}$ for $j_{x}=0$ inside the rectangle implies $r_{1}=r_{2}=b_{0} m_{y}$, i.e., $c_{X}=0, c_{Y}=c_{0}$." Both Eqs. (17) and (18) contain the wrong signs in $e^{-b_{0} m_{x} L_{y}}$ in the denominators. So, Eq. (17) should read

$$
\mu(x, y)=-\frac{I_{\mathrm{c}}}{\sigma^{\prime}} \frac{b_{0} m_{y} e^{b_{0} m_{y} x}}{e^{b_{0} m_{y} L_{x}}-1} \frac{e^{-b_{0} m_{x} y} \cosh \left(c_{0} y\right)}{e^{-b_{0} m_{x} L_{y}} c_{0} \sinh \left(c_{0} L_{y}\right)},
$$

and Eq. (18) should read

$$
\mu(x, y)=-\frac{I_{\mathrm{c}}}{\sigma^{\prime}} \frac{1}{L_{x}} \frac{e^{-b_{0} m_{x} y} \cosh \left(c_{0} y\right)}{e^{-b_{0} m_{x} L_{y}} c_{0} \sinh \left(c_{0} L_{y}\right)} .
$$

All the above corrections do not affect the results, the discussion, and the conclusion of the original article. 